\title{
Energy spectra of a particle confined in a finite ellipsoidal shaped potential well
}

Tamaz Kereselidze ${ }^{\mathrm{a}}$, Tamar Tchelidze ${ }^{\mathrm{a}}$, Teimuraz Nadareishvilia ${ }^{\mathrm{a}}$, Roman Ya. Kezerashvili ${ }^{\mathrm{b}, \mathrm{c}}$

${ }^{a}$ Faculty of Exact and Natural Sciences, Tbilisi State University, 0179 Tbilisi, Georgia,

${ }^{\mathrm{b}}$ Physics Department, New York City College of Technology, The City University of New York, Brooklyn, NY 11201 USA

${ }^{c}$ Graduate School and University Center, The City University of New York, NY 10016, USA

\begin{abstract}
A charged particle confined in a strongly prolate ellipsoidal shaped finite potential well is studied. In the case when a distance $R$ between foci is large and accordingly $R^{-1}$ is small, the asymptotic solutions of quasiradial and quasiangular equations in prolate spheroidal coordinates are found. We demonstrate that quasiangular wave functions inside and outside of the potential well coincide on the entire surface of strongly prolate ellipsoid if separation parameters are chosen appropriately. This allows us to obtain the transcendental equation for the energy levels by equating the quasiradial wave function and its derivative on the surface of ellipsoid.

The obtained equation is solved numerically and algebraically. The calculated energies are in good qualitative and quantitative agreement with the results obtained earlier for the infinitely high ellipsoidal potential well via a numerical solution of the quasiradial and quasiangular equations. An importance of the actual shape of ellipsoidal potential well for calculation of the energy spectrum for the trapped particle is shown. A dependence of the energy spectrum on the effective mass when it is a different constant inside and outside of the ellipsoid is addressed.
\end{abstract}

Keywords: Nanoparticle, Ellipsoidal shape, Energy levels, Prolate spheroidal coordinates

\section{Introduction}

Interest is rapidly growing in crystalline semiconductor structures of various shapes and nanometer size. The physical properties of objects such as nanorods, nanowires and quantum dots have been intensively studied both theoretically and experimentally [1]. The most important result revealed in these investigations is the strong interdependence between the character of the energy spectrum of a nanosize object and its geometrical parameters [2-7].

The geometrical parameters of nanoparticles depend strongly on the nature of their production. At the growing of semiconductor nanoparticles by various methods, the energy spectrum varies continuously with the size and shape of nanoparticle. The experiments indicate that small nanoparticles have a nearly spherical shape, whereas large nanoparticles have an ellipsoidal shape [8]. Both experimental data and numerical simulations show that the shape of nanoparticles typically exhibit a transformation from the spherical to the ellipsoidal shape and the effect of shape transformation is important. Ellipsoidal nanoparticles play an important role for applications and one of the most promising candidates for technological applications is just the ellipsoidal prolonged nanoparticles. Thus, knowledge of the electronic and optical properties of ellipsoidal nanoparticles is 
crucial for further technological advances. In the regime of strong size quantization, the electron-hole Coulomb interaction energy is much less than the confinement energy. Hence, in this regime one can neglect the Coulomb interaction and treat the electron and the hole independently. Calculation of the energy spectra and appropriate wave functions for nanoparticles of an ellipsoidal shape is a complicated task even in a one-particle approximation. The problem becomes solvable in two limiting cases: for nanoparticles of nearly spherical shape [9-12] and for nanoparticles of strongly prolate ellipsoidal shape [1316]. In these cases the problem involves a small parameter that can be used for an approximate solution.

In preceding works [13-15], the authors assumed that a charged particle is mainly localized in the domain near the origin and the problem was treated in cylindrical coordinates for a nanoparticle that has an ellipsoidal shape. As a result the obtained energy spectrum for an infinite potential well has a family of equidistant levels.

The effective-mass Schrödinger equation for an electron trapped in the ellipsoidal shape infinitely high potential well was separated in spheroidal coordinates and the obtained system of coupled equations was solved numerically [17-20]. An elaborated scheme of calculation allowed the authors to calculate the energy levels of ellipsoidal nanoparticles for diverse shapes of an ellipsoid [19]. An analysis of those results at a large values of the parameter $c / a$ ( $a$ and $c$ are a prolate ellipsoidal semi-axes) that demonstrates the deviation from a spheroid, shows that the energy levels corresponding to the motion along the ellipsoidal axis are not equidistant.

We recently considered a charged particle confined in a potential well that has almost spherical shape and infinitely high wall [21]. In this case the parameter $c / a$ is small and the problem is accordingly solvable within perturbation theory. Explicit expressions for the energy levels and the corresponding wave functions were obtained in the third and second orders of perturbation approximation, respectively. The problem was also solved for arbitrary $c / a$ of the ellipsoidal potential well using the Hamiltonian diagonalization method on the basis of wave functions corresponding to the spherical potential well. The obtained results exhibit perfect agreement with preceding results [19], and confirm that the energy levels corresponding to the motion along the ellipsoid axis are not equidistant.

The problem of the motion of a charged particle in a potential well of finite depth is theoretically much more complicated [22-24]. In this case the traditional method of obtaining eigenvalues on matching the wave function and its derivative at the boundary of the ellipsoid does not work [23, 24]. A justification of this statement is that the equation providing the continuity of the total wave function, which is a product of the quasiradial and quasiangular functions, cannot be satisfied for an arbitrary quasiangular variable [23].

A large number of important physical problems require solving the Schrödinger equation for potential of different symmetry to determine the eigenfunctions and eigenvalues. The Schrödinger equation is analytically exactly solvable for very limited potentials. In general one has an option to use numerical techniques or approximation methods like perturbation theory, WKB approximation, asymptotic method and etc. for the solution. We note that, for a nanorod of cylindrical shape the problem of finding energy spectrum can have an exact solution when one assumes that the potential experienced by a charged particle is zero inside a cylinder and an infinite [25] or a finite constant [26] on the surface of cylinder and outside.

The aim of the present work is to elaborate asymptotic method for finding both the eigenfunctions and eigenvalues of the Schrödinger equation for a strongly prolate ellipsoidal shaped finite potential well. Our purpose is twofold: firstly, to demonstrate that the energy levels corresponding to the motion along the ellipsoid axis are not equidistant when the actual shape of ellipsoidal nanoparticle is taken into account; secondly, to show that the method of obtaining eigenvalues on matching the wave function and its derivative is valid when the potential well has a strongly prolate ellipsoidal shape and a finite depth. We demonstrate that, 
for a strongly prolate ellipsoidal potential well, the inside and outside quasiangular wave functions coincide on the surface of ellipsoid within the accuracy of the leading term, when the separation parameter is chosen appropriately. A coincidence of the quasiangular wave functions allows obtaining the energy levels of a charged particle by matching the quasiradial wave function and its derivative on the surface of ellipsoid.

The paper is organized as follows. In Section 2, the basic equations are given in prolate spheroidal coordinates. In Section 3, we treat a charged particle confined in the potential well of a strongly prolate ellipsoidal shape and derive a transcendental equation for the energy levels. In Section 4, we discuss the effective-mass approximation. We present the results of our calculations and draw conclusions in Sections 5 and 6, respectively.

\section{Particle in the prolate ellipsoidal potential well}

We start our study with a standard quantum mechanics approach for a particle in a finite potential well and a solution is required to satisfy the standard condition: continuity of the wave function and its derivative [27]. We consider the particle of mass $m_{0}$ in a finite potential well which has prolate ellipsoidal shape with semi-axes $a$ and $c$, where the parameter $a$ represents the size of a well in the $x$ and $y$ directions and $c$ is the size in the $z$ direction. In orthogonal Cartesian coordinates the surface of ellipsoid is determined by equation

$$
\frac{x^{2}+y^{2}}{a^{2}}+\frac{z^{2}}{c^{2}}=1
$$

The simplest potential that confines a charged particle in an ellipsoid is zero inside of the ellipsoid, it is finite outside and on the surface of the ellipsoid and has the form

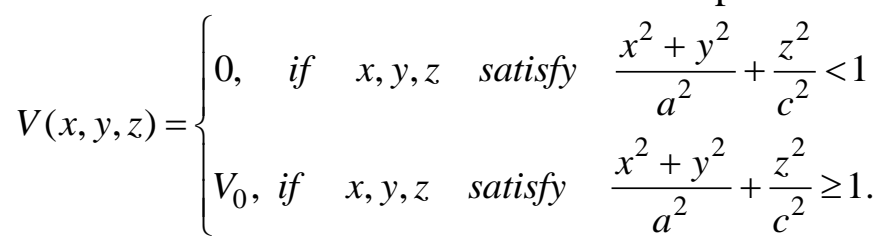

The corresponding Schrödinger equation with potential (2) that describes the motion of a trapped particle reads

$$
\left(-\frac{\hbar^{2}}{2 m_{0}} \Delta_{x y z}+V(x, y, z)\right) \Psi(x, y, z)=E \Psi(x, y, z)
$$

where $\Delta_{x y z}$ is the Laplace operator with respect to the variables $x, y, z$ and $E$ is particle's energy that is measured from the bottom of the well. Eq. (3) can be rewritten in the following form:

$$
\left(\Delta_{x y z}+\kappa^{2}\right) \Psi(x, y, z)=0,
$$

where $\kappa=k=\sqrt{2 m_{0} E / \hbar^{2}}$ when a charged particle is inside of the ellipsoid, and $\kappa=i \alpha$ with $\alpha=\sqrt{\beta^{2}-k^{2}}$ and $\beta=\sqrt{2 m_{0} V_{0} / \hbar^{2}}$ when a particle is on the surface of ellipsoid and outside of it.

Due to the symmetry of the system, for the solution of Eq. (4) it is appropriate to introduce prolate spheroidal coordinates $\xi, \eta, \varphi$ where $1 \leq \xi<\infty,-1 \leq \eta \leq 1,0 \leq \varphi \leq 2 \pi$. This set of coordinates is related to the Cartesian coordinates as follows:

$$
\begin{aligned}
& x=\frac{R}{2} \sqrt{\left(\xi^{2}-1\right)\left(1-\eta^{2}\right)} \cos \varphi, \\
& y=\frac{R}{2} \sqrt{\left(\xi^{2}-1\right)\left(1-\eta^{2}\right)} \sin \varphi,
\end{aligned}
$$




$$
z=\frac{R}{2} \xi \eta
$$

where $R$ is the confocal distance along axis $z$.

In prolate spheroidal coordinates (1) can be written as

$$
\frac{\left(\xi^{2}-1\right)\left(1-\eta^{2}\right)}{a^{2}}+\frac{\xi^{2} \eta^{2}}{c^{2}}=\frac{4}{R^{2}} .
$$

By putting in (6) $\eta=\eta_{0}$ where $-1 \leq \eta_{0} \leq 1$ and taking into account that the small and large semi-axes are bounded with a condition $4\left(c^{2}-a^{2}\right)=R^{2}$, we find that $\xi$ becomes $\xi^{*}=\left(1-a^{2} / c^{2}\right)^{-1 / 2}$ on the surface of ellipsoid. Thus on the surface of ellipsoid $\xi=\xi^{*}$, whereas $\eta$ can take any value between -1 and +1 .

Writing the Laplace operator in Eq. 4 in prolate spheroidal coordinates and representing the wave function as a product of three functions $\Psi=X(\xi) Y(\eta) e^{ \pm i m \varphi}$ with $m$ as the modulus of the magnetic quantum number, one can get the following coupled equations:

$$
\begin{aligned}
& \frac{d}{d \xi}\left(\xi^{2}-1\right) \frac{d X(\xi)}{d \xi}+\left[\lambda+\frac{\kappa^{2} R^{2}}{4}\left(\xi^{2}-1\right)-\frac{m^{2}}{\xi^{2}-1}\right] X(\xi)=0, \\
& \frac{d}{d \eta}\left(1-\eta^{2}\right) \frac{d Y(\eta)}{d \eta}+\left[-\lambda+\frac{\kappa^{2} R^{2}}{4}\left(1-\eta^{2}\right)-\frac{m^{2}}{1-\eta^{2}}\right] Y(\eta)=0,
\end{aligned}
$$

where $X(\xi)$ and $Y(\eta)$ denote the quasiradial and quasiangular wave functions by analogy to a spherical quantum dot [1] and $\lambda$ is the separation parameter. At the first view these equations are formally equivalent and should have the same solutions. However, it is not so because they must be solved in different range of corresponding variables $\xi$ and $\eta$ : one is looking for the solution of Eq. (7a) for $1 \leq \xi<\infty$, while Eq. (7b) should be solved in the range of $-1 \leq \eta \leq 1$.

To find the asymptotic solutions of Eqs. (7a) and (7b), usually instead of functions $X(\xi)$ and $Y(\eta)$ new functions are introduced

$$
\begin{aligned}
& U(\xi)=\left(\xi^{2}-1\right)^{1 / 2} X(\xi), \\
& V(\eta)=\left(1-\eta^{2}\right)^{1 / 2} Y(\eta) .
\end{aligned}
$$

These functions must satisfy the boundary conditions $U(1)=0$ and $V( \pm 1)=0$, which provide the condition for the wave functions $X(\xi)$ and $Y(\eta)$ to be finite at $\xi=1$ and $\eta= \pm 1$.

Substituting (8) into (7), one obtains the following equations for unknown functions $U(\xi)$ and $V(\eta)$ :

$$
\begin{aligned}
& \frac{d^{2} U_{i n s}(\xi)}{d \xi^{2}}+\left[\frac{k^{2} R^{2}}{4}+\frac{\lambda_{i n}}{\xi^{2}-1}+\frac{1-m^{2}}{\left(\xi^{2}-1\right)^{2}}\right] U_{i n s}(\xi)=0, \\
& \frac{d^{2} V_{i n s}(\eta)}{d \eta^{2}}+\left[\frac{k^{2} R^{2}}{4}-\frac{\lambda_{i n}}{1-\eta^{2}}+\frac{1-m^{2}}{\left(1-\eta^{2}\right)^{2}}\right] V_{i n s}(\eta)=0,
\end{aligned}
$$

when a charged particle is inside of the ellipsoid, and

$$
\begin{aligned}
& \frac{d^{2} U_{\text {out }}(\xi)}{d \xi^{2}}+\left[-\frac{\alpha^{2} R^{2}}{4}+\frac{\lambda_{\text {out }}}{\xi^{2}-1}+\frac{1-m^{2}}{\left(\xi^{2}-1\right)^{2}}\right] U_{\text {out }}(\xi)=0, \\
& \frac{d^{2} V_{\text {out }}(\eta)}{d \eta^{2}}+\left[-\frac{\alpha^{2} R^{2}}{4}-\frac{\lambda_{\text {out }}}{1-\eta^{2}}+\frac{1-m^{2}}{\left(1-\eta^{2}\right)^{2}}\right] V_{\text {out }}(\eta)=0,
\end{aligned}
$$

when a charged particle is on the surface and outside of the ellipsoid. From (9) and (10) follows that these equations have the first and the second order irregularities at $\xi=1$ and $\eta= \pm 1$. Another peculiarity of these equations is that (9a), (9b) and (10a), (10b) are formally 
identical equations but have distinct domains for the quasiradial $\xi$ and quasiangular $\eta$ variables.

\section{Asymptotically exact solutions}

Let us consider a prolate ellipsoid with semi-axes of length $a$ and $c$ inscribed in a cylinder of the length $L=2 c$ and radius $a$ as shown in Fig 1. Away from the foci the shape of prolate ellipsoid resembles the shape of cylinder. Obviously, there should be similarity between the solutions of the Schrödinger equation for a prolate ellipsoidal and a cylindrical potential well for a large $c / a$.

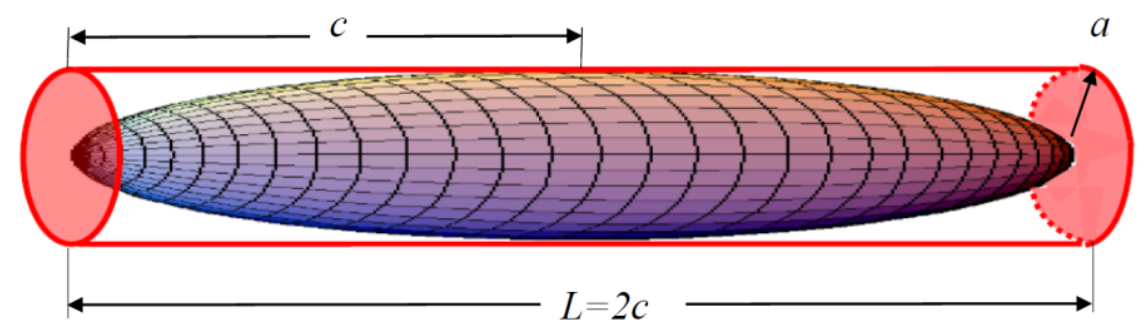

Fig. 1. Ellipsoid with semi-axes $a$ and $c$ is inscribed into a cylinder of length $L=2 c$ and radius $a$.

When the potential well has a strongly prolate ellipsoidal shape, i.e. $c>>a$, the confocal distance $R$ in Eqs. (9) and (10), has a large value of $R=2 \sqrt{c^{2}-a^{2}}>1$. The inverse value $R^{-1}$ is accordingly small. Therefore, one can use this parameter to find the asymptotic solutions of Eqs. (9) and (10). Our scheme of calculation is to determine the separation parameter $\lambda_{\text {in }}$ in Eq. (9b) by employing a similarity with the equation that describes the parallel motion in a cylindrical potential well. For this purpose in Appendix A the sketch of solution for a charged particle confined in a cylindrical potential well is presented. Thereafter we demonstrate that the solutions of quasiangular Eqs. (9b) and (10b) coincide on the surface of ellipsoid when separation parameter $\lambda_{\text {out }}$ is appropriately related to $\lambda_{\text {in }}$. We solve the quasiradial Eqs. (9a) and (10a) using the obtained separation parameters $\lambda_{\text {in }}$ and $\lambda_{\text {out }}$, and by equating the inside and outside quasiradial wave functions and their derivatives on the surface of ellipsoid, find the energy spectrum for a charged particle.

\subsection{Quasi-angular solutions}

As it was mentioned above away from the foci the shape of strongly prolate ellipsoidal potential well resembles the shape of cylindrical potential well. In the domain $|\eta|<<1$, the solutions of Eqs. (9b) and (A.1) are expected to be similar. To show this similarity, we introduce a new variable $z=R \xi^{*} \eta / 2$ and using the identical transformation (B.1) presented in Appendix B, we write Eq. (9b) as

$$
\frac{d^{2} V_{i n}}{d z^{2}}+\left[p_{i n}^{2}+v_{i n}(z)\right] V_{i n}=0,
$$

where

$$
p_{i n}^{2}=\left(k^{2}-4 \lambda_{\text {in }} / R^{2}\right) / \xi^{* 2},
$$




$$
v_{i n}=-\frac{16 z^{2}}{R^{4} \xi^{* 4}} \frac{\lambda_{\text {in }}}{\left(1-4 z^{2} / R^{2} \xi^{* 2}\right)}+\frac{4\left(1-m^{2}\right)}{R^{2} \xi^{* 2}\left(1-4 z^{2} / R^{2} \xi^{* 2}\right)^{2}} .
$$

In the domain $|z|<R^{1 / 2}$, i.e. in the region of ellipsoid where $\xi \sim 1$ and $|\eta|<R^{-1 / 2}<<1$, $v(z)$ has a magnitude of order $R^{-1}$ or less (as will be shown, $\lambda_{\text {ins }}$ has a magnitude of order $\left.R^{2}\right)$. Therefore, $v(z)$ can be neglected in (11). The residual equation has solutions $\cos (p z)$ and $\sin (p z)$, which must coincide with the solutions of Eq. (A.1) $\cos \left(k_{z} z\right)$ and $\sin \left(k_{z} z\right)$ that describe the motion along axis $z$ in the cylindrical well. These conditions determine the separation parameter $\lambda_{i n}$. After performing the appropriate calculation, one obtains

$$
\lambda_{i n}^{e v, o d}=\frac{R^{2}}{4}\left[k^{2}-\left(\frac{t_{n_{e v, o d}}}{c}\right)^{2}\right]+O\left(R^{-1}\right) .
$$

In Eq. (12) $\lambda_{\text {in }}^{e v}$ corresponds to even and $\lambda_{\text {in }}^{\text {od }}$ to odd states with respect to replacement $\eta \rightarrow-\eta$ or alternatively $z \rightarrow-z$, while $t_{n_{e v}}$ and $t_{n_{o d}}$ denote the roots of transcendental Eqs. (A.5) and (A.6) presented in Appendix A. Since the separation parameter does not depend on the variables, the expression derived for $\lambda_{i n}^{e v, o d}$ can be used to find the solutions of Eqs. (9a) and (9b) in the entire domain of the variables, including the critical regions near the foci.

In the domain near the left focus, $(1+\eta)$ is small. Introducing a new variable $r_{1}=i k R(1+\eta)$, Eq. (9b) can be written as

$$
\frac{d^{2} V_{i n}}{d r_{1}^{2}}+\left[-\frac{1}{4}-\frac{\lambda_{i n}}{2 i k R r_{1}\left(1-r_{1} / 2 i k R\right)}+\frac{1-m^{2}}{4 r_{1}^{2}\left(1-r_{1} / 2 i k R\right)^{2}}\right] V_{i n s}=0,
$$

where $\lambda_{i n}$ is defined by (12). Here and below, we omit the superscripts in the wave functions and separation parameters.

Using the identical transformations (B.1) and (B.2) in Eq. (13), one obtains

$$
\frac{d^{2} V_{i n}}{d r_{1}^{2}}+\left[-\frac{\gamma_{i n}^{2}}{4}-\frac{\lambda_{i n}}{2 i k R r_{1}}+\frac{1-m^{2}}{4 r_{1}^{2}}+u_{i n}\right] V_{i n}=0,
$$

where $\gamma_{\text {in }}^{2}=1-\lambda_{\text {in }} /\left(k^{2} R^{2}\right)$ and

$$
u_{i n}=\frac{\lambda_{i n}}{4 k^{2} R^{2}} \frac{r_{1} / 2 i k R}{1-r_{1} / 2 i k R}+\frac{1-m^{2}}{4 r_{1}^{2}} \frac{r_{1} / i k R-\left(r_{1} / 2 i k R\right)^{2}}{\left(1-r_{1} / 2 i k R\right)^{2}} .
$$

Eq. (14) is equivalent to Eqs. (13) and (9b). The last term in the squared brackets has a magnitude of order $R^{-1}$ in the domain near the left focus. Therefore, $u_{\text {in }}$ can be neglected in the region where $r_{1}<1$. The solution of the residual equation, which satisfies the necessary boundary condition at $r_{1}=0$, is the Whittaker function $M_{\mu, m / 2}\left(\gamma_{i n} r_{1}\right)$ with $\mu=-\lambda_{i n} /\left(2 i k R \gamma_{i n}\right)$ [28]. Returning to the variable $\eta$, we thus write $V_{i n}=M_{\mu, m / 2}\left(i k R \gamma_{i n}(1+\eta)\right)$ when $\eta \sim-1$. Near the right focus, i.e. in the domain where $(1-\eta)$ is small, the solution of Eq. (9b) is $V_{i n}=M_{\mu, m / 2}\left(\gamma_{i n} r_{2}\right)$ with $r_{2}=i k R(1-\eta)$.

In the wave functions $V_{i n}=M_{\mu, m / 2}\left(\gamma_{i n} r_{1,2}\right)$ the variable $\gamma_{i n s} r_{1,2}<1$, whereas the first parameter is large $(\mu \sim R>>1)$. This fact allows us to use the asymptotic form for the Whitaker function with respect to the first parameter $\left(M_{\mu, m / 2}(x) \rightarrow m ! \mu^{-m / 2} x^{1 / 2} J_{m}(2 \sqrt{\mu x})\right.$ if $\mu x$ is restricted and $\mu \rightarrow \infty$ [29]). In consequence, we obtain

$$
V_{i n}=m ! \mu^{-m / 2}\left[i k \gamma_{i n} R(1 \pm \eta)\right]^{1 / 2} J_{m}\left(\sqrt{-2 \lambda_{i n}(1 \pm \eta)}\right)+O\left(R^{-1}\right)
$$


where $J_{m}(x)$ is the Bessel function of the first kind.

The desired solutions of Eq. (10b) near the left and right foci can be obtained in the same way as $V_{i n}$, which yields

$$
V_{\text {out }}=M_{\mu, m / 2}\left(\alpha R \gamma_{\text {out }}(1 \pm \eta)\right) \text {. }
$$

In (16) $\mu=-\lambda_{\text {out }} /\left(2 \alpha R \gamma_{\text {out }}\right), \gamma_{\text {out }}=\left(1+\lambda_{\text {out }} /\left(\alpha^{2} R^{2}\right)\right)^{1 / 2}$ and signs \pm correspond to the left and right foci, respectively.

For $x<<1$ the Bessel function $J_{m}(x) \sim(m !)^{-1}(x / 2)^{m}$ and the Whittaker function $M_{\mu, m / 2}(x) \sim x^{(m+1) / 2}$ [28]. Taking these conditions into account, one can readily show that the wave functions (15) and (16) coincide when the condition $i k \gamma_{i n s}=\alpha \gamma_{o u t}$ is satisfied. This condition determines the separation parameter $\lambda_{\text {out }}$, which is

$$
\lambda_{\text {out }}=\lambda_{\text {in }}-\beta^{2} R^{2},
$$

where $\lambda_{\text {in }}$ is defined by Eq. (12) and $\beta^{2}=2 m_{0} V_{0} / \hbar^{2}$. Thus, we obtain that the inside and outside quasiangular wave functions coincide in the domains near the left and right foci when condition (17) is fulfilled. Substituting (17) into $\gamma_{\text {out }}=\left(1+\lambda_{\text {out }} /\left(\alpha^{2} R^{2}\right)\right)^{1 / 2}$ and taking into account that $\beta^{2}>>k^{2}$, we obtain $\gamma_{\text {out }}=i \sqrt{3} /(2 \beta)$. So that $\gamma_{\text {out }}$ is purely imaginary value, which is in consistent with equation $\gamma_{\text {out }}=i k \gamma_{\text {in }} / \alpha$.

Introducing a new variable $z=(\eta-\varepsilon) R(9 \mathrm{~b})$ and (10b) can be written as

$$
\begin{aligned}
& \frac{d^{2} V_{\text {in }}}{d z^{2}}+\left[\frac{k^{2}}{4}-\frac{\lambda_{\text {in }}}{R^{2}\left(1-(\varepsilon+z / R)^{2}\right)}+\frac{1-m^{2}}{R^{2}\left(1-(\varepsilon+z / R)^{2}\right)^{2}}\right] V_{\text {in }}=0, \\
& \frac{d^{2} V_{\text {out }}}{d z^{2}}+\left[-\frac{\alpha^{2}}{4}-\frac{\lambda_{\text {out }}}{R^{2}\left(1-(\varepsilon+z / R)^{2}\right)}+\frac{1-m^{2}}{R^{2}\left(1-(\varepsilon+z / R)^{2}\right)^{2}}\right] V_{\text {out }}=0 .
\end{aligned}
$$

The solutions of Eqs. (18a) and (18b) coincide in the region $z<<R(\eta \sim 0)$ if the condition $\lambda_{\text {out }}=\lambda_{\text {in }}-\beta^{2} R^{2}\left(1-\varepsilon^{2}\right) / 4$ if fulfilled. Assuming that $\varepsilon=i \sqrt{3}$, we thereby come to (17).

Thus, we have shown that the inside and outside quasiangular wave functions coincide within the accuracy of the leading term on the entire surface of ellipsoid ( $\xi=\xi^{*}$, $-1 \leq \eta \leq 1)$

$$
V_{\text {in }}\left(\eta, \lambda_{\text {in }}\right)=V_{\text {out }}\left(\eta, \lambda_{\text {out }}\right)+O\left(R^{-1}\right),
$$

when the separation parameters $\lambda_{\text {in }}$ and $\lambda_{\text {out }}$ are bound by (17). At $R \rightarrow \infty$ there is a situation analogous to the spherical quantum dot $(R=0)$ with a finite depth. In that case, the inside and outside angular wave functions are identical and canceled out. So that matching of the radial wave functions and their derivatives on the surface of sphere leads to the energy eigenvalues.

\subsection{Quasi-radial solutions}

Now we solve the quasiradial Eqs. (9a) and (10a) using the derived separation parameters (12) and (17). Introducing a new variable $r=i k R(\xi-1)$, Eq. (9a) can be written as

$$
\frac{d^{2} U_{i n}}{d r^{2}}+\left[-\frac{1}{4}+\frac{\lambda_{i n}}{2 i k R r(1+r / 2 i k R)}+\frac{1-m^{2}}{4 r^{2}(1+r / 2 i k R)^{2}}\right] U_{i n}=0
$$


which determines the quasiradial wave function inside the ellipsoid.

In Eq. (20) the term $r / 2 i k R$ is small and hence can be neglected. The solution of the residual equation, which satisfies the necessary boundary condition at $r=0$, is the Whittaker function $M_{\mu, m / 2}(r)$ with $\mu=\lambda_{i n} /(2 i k R)$ [28]. Returning to the variable $\xi$, for the solution of Eq. (9a) one can get

$$
U_{i n}(\xi)=M_{\mu, m / 2}(i k R(\xi-1))+O\left(R^{-1}\right) .
$$

Using an asymptotic form of the Whittaker function with respect to the first parameter [29], one obtains for the quasiradial wave function inside of the ellipsoid

$$
U_{i n}=m ! \mu^{-m / 2}[i k R(\xi-1)]^{1 / 2} J_{m}\left(\sqrt{2 \lambda_{i n}(\xi-1)}\right)+O\left(R^{-1}\right) .
$$

In (10a) that describes the motion of a charged particle on the surface and outside of the ellipsoid, we introduce a new variable $r=\alpha R(\xi-1)$, and write

$$
\frac{d^{2} U_{\text {out }}}{d r^{2}}+\left[-\frac{1}{4}+\frac{\lambda_{\text {out }}}{2 \alpha R r(1+r / 2 \alpha R)}+\frac{1-m^{2}}{4 r^{2}(1+r / 2 \alpha R)^{2}}\right] U_{\text {out }}=0,
$$

Within the accuracy of the leading term, the solution of (23) is the Whittaker function $W_{\mu, m / 2}(r)$ which is regular at $r \rightarrow \infty$ [28]. Therefore, we obtain

$$
U_{\text {out }}=W_{\mu, m / 2}(\alpha R(\xi-1))+O\left(R^{-1}\right),
$$

with $\mu=\lambda_{\text {out }} /(2 \alpha R)$.

\subsection{Energy levels}

For the infinite potential well the energy levels can be readily obtained by equating the quasiradial wave function (22) to zero on the surface of ellipsoid. Indeed, $J_{m}\left(\sqrt{2 \lambda_{\text {in }}\left(\xi^{*}-1\right)}\right)=0$ gives

$$
k_{0}=\left(\frac{2 \tau_{s, m}^{2}}{R^{2}\left(\xi^{*}-1\right)}+\frac{\pi^{2} n_{e v, o d}^{2}}{4 c^{2}}\right)^{1 / 2},
$$

which through the equation $E=\hbar^{2} k^{2} / 2 m_{0}$ allows us to write [16]

$$
E_{s m n_{e v, o d}}^{0}=\frac{\hbar^{2}}{2 m_{0}}\left[\frac{\tau_{s, m}^{2}}{2\left(c \sqrt{c^{2}-a^{2}}-\left(c^{2}-a^{2}\right)\right)}+\left(\frac{\pi n_{e v, o d}}{2 c}\right)^{2}\right] .
$$

In (26) $\tau_{s, m}$ is the dual-index $s^{\text {th }}$ root of the Bessel function and $n_{e v}=1,3,5, \ldots$ for the even and $n_{o d}=2,4,6, \ldots$ for the odd states, respectively.

In case of the finite potential well, we equate the quasiradial wave functions (22) and (24) and their derivatives on the surface of the ellipsoid and obtain an equation that determines $k^{2}$ and accordingly, the eigenenergy of a charged particle

$$
\left(\frac{1}{J_{m}} \frac{d J_{m}}{d \xi}+\frac{1}{2(\xi-1)}\right)_{\xi=\xi^{*}}=\left(\frac{1}{W_{\mu, m / 2}} \frac{d W_{\mu, m / 2}}{d \xi}\right)_{\xi=\xi^{*}} .
$$

Representing $d J_{m} / d \xi$ and $d W / d \xi$ through the Bessel and Whittaker functions [28], respectively, (27) readily reduces to the equation

$$
\left[\frac{x^{2} J_{m}(x)}{x J_{m+1}(x)-m J_{m}(x)}\right]_{x=\sqrt{2 \lambda_{i n}\left(\xi^{*}-1\right)}}=\frac{2 \lambda_{i n}}{\alpha R}\left[1-\frac{m}{y}+\chi_{\mu, m / 2}(y)\right]_{y=\alpha R\left(\xi^{*}-1\right)}^{-1},
$$

where 


$$
\chi_{\mu, m / 2}(y)=\frac{(m+1-2 \mu)}{y^{1 / 2}} \frac{W_{\mu-1 / 2,(m+1) / 2}(y)}{W_{\mu, m / 2}(y)} .
$$

Eq. (28) is a transcendental equation and hence can be solved only numerically. However, it also allows obtaining an approximate algebraic solution.

Let's obtain the approximate algebraic solution of Eq. (28). When the potential is high but finite, the algebraic solution of Eq. (28) can be found by substituting in (28) $k=k_{0}+\Delta k$ and assuming that $\Delta k$ is a small correction. Using Eq. (A.7) for $t_{n_{e v, o d}}$, one can write that $\Delta k=-2^{-1} k_{0} \Omega V_{0}^{-1 / 2}$ in which

$$
\Omega=\sqrt{\frac{2}{m_{0}}} \frac{\hbar}{k_{0}^{2}}\left[\frac{2 \tau_{s, m}^{2}}{R^{3}\left(\xi^{*}-1\right)^{2}}\left(1+\chi_{\mu_{0}, m / 2}\left(y_{0}\right)\right)^{-1}+\frac{\pi^{2} n_{e v, o d}^{2}}{2^{3 / 2} c^{3}}\right],
$$

$\mu_{0}=-\beta R / 2$ and $y_{0}=\beta R\left(\xi^{*}-1\right)$. For the energy spectrum, we thus obtain

$$
E_{s m n_{e v, o d}}=E_{s m n_{e v, o d}}^{0}\left(1-\frac{\Omega_{s m n_{e v, o d}}}{\sqrt{V_{0}}}\right) \text {, }
$$

where $E_{s m n_{e v, o d}}^{0}$ and $\Omega_{s m n_{e v, o d}}$ are defined with Eqs. (26) and (30).

The Eqs. (26) and (31) for the energy spectrum show that each level corresponding to the motion perpendicular to the ellipsoid axis has its own family of levels. These levels are related to the parallel motion along the ellipsoid axis and are not equidistant. Eq. (26) transforms into the expression for the energy spectrum of a cylindrical shaped nanorod and the infinite potential well (Eq. (A.10) in Appendix A), when it is expanded in terms of a small value $a / c$ and terms proportional to $(a / c)^{2}$ and less are neglected. We note that $\Omega$ depends on $V_{0}$ through $\chi_{\mu_{0}, m / 2}\left(y_{0}\right)$, and but this dependence is weak. Hence it should be expected that the energy levels are linear functions of $V_{0}^{-1 / 2}$ in the region of validity of (31), i.e. at small $V_{0}^{-1 / 2}$.

We proceed to answer the question what kind of approximation should be made in the approach developed above to obtain equidistant energy levels? For this purpose, we assume that a charged particle is localized in the vicinity of the point $\eta=0$ or $z=0$. This assumption enables us to expand $v(z)$ in power of $z^{2}$ and to retain only the first term in this expansion. The solution of Eq. (11) with $v=-\Omega z^{2}$ where $\Omega=16 \lambda /\left(R \xi^{*}\right)^{4}$ is represented with Hermit polynomials when $\lambda=R^{2}\left(k^{2}-\xi^{*^{2}}(2 n+1)\right) / 4$ and $n=0,1,2 \ldots$ [27]. Employing the derived separation parameter and making the calculations analogous to that made in (20)-(26), we obtain for the energy spectrum for an infinite potential well

$$
E_{s m n}=\frac{\hbar^{2}}{2 m_{0}}\left[\frac{\tau_{s, m}^{2}}{a^{2}}+\frac{2 \tau_{s, m}}{a c}\left(n+\frac{1}{2}\right)\right] .
$$

In (32) $\tau_{s, m}$ is the $s^{\text {th }}$ root of the Bessel function of the first kind and $n=0,1,2, \ldots$. This expression coincides with the analogous one obtained in [13-15] using a parabolic approximation for the effective potential related to the parallel motion. The representation of $v(z)$ as a parabolic potential hence leads us to the equidistant energy levels and is incorrect. Accordingly is incorrect an assumption that a charged particle is localized in the vicinity of the point $z=0$. 


\section{Effective-mass approximation}

A charged particle in a quantum well assumed to have an effective mass, which depends on the material making up the well. When this material is embedded in some other material to make a trap quantum mechanics predicts that the charged particle can penetrate into trapped material where it has a different value for the effective mass. Usually in such heretostructures the effective mass in general is position dependent. In our case assuming that the effective mass is a different constant inside and outside of the ellipsoid, one can write $m_{0}=m_{\text {in }}+\left(m_{\text {out }}-m_{\text {in }}\right) \theta\left(\xi-\xi^{*}\right)$, where the step function $\theta\left(\xi-\xi^{*}\right)$ is defined as $\theta\left(\xi-\xi^{*}\right)=0$, when $\xi-\xi^{*}<0$ and $\theta\left(\xi-\xi^{*}\right)=1$, when $\xi-\xi^{*} \geq 0$.

Our model considered above has some limitation related to its application to semiconductors. A quantum well produces by a semiconductor interface is more subtle than an ideal potential well considered above. Particularly, one has to know what is the matching condition for the wave function at a material interface. If we consider Eq. (3) as the effective-mass Schrödinger equation for an envelope function with potential (2) that describes the motion of a trapped particle, this equation will have a different effective mass for in- and out-side of the ellipsoid: $m_{0}=m_{\text {in }}$ and $m_{0}=m_{\text {out }}$. One wants to know what should be the matching conditions in this case. Various prescriptions have been proposed (see, for example, review [30]) for the matching conditions of the wave function and its derivative at a semiconductor interface to calculate confined-state energies, but situation remains still controversial [31,32]. All possible conditions ensure that the solution for the energy should be real, however, the conditions for the continuity of the envelope wave function and matching the ratio of its derivative and the effective mass inside and outside of the trap (this ratio should be continuous) is favored because it also ensures continuity of the probability current. This condition is known as the BanDaniel-Duke-Bastard boundary conditions $[33,34]$ in the context of semiconductor heterostructures. Using this condition in the effective mass approximation Eq. (27) reduces to

$$
\frac{1}{m_{\text {in }}}\left(\frac{1}{J_{m}} \frac{d J_{m}}{d \xi}+\frac{1}{2(\xi-1)}\right)_{\xi=\xi^{*}, m_{0}=m_{\text {in }}}=\frac{1}{m_{\text {out }}}\left(\frac{1}{W_{\mu, m / 2}} \frac{d W_{\mu, m / 2}}{d \xi}\right)_{\xi=\xi^{*}, m_{0}=m_{0 u t}} .
$$

Using condition (33) and expressing derivatives $d J_{m} / d \xi$ and $d W / d \xi$ through the Bessel and Whittaker functions [28] one obtains the transcendental equation that determines $k^{2}$ and accordingly, the eigenenergy of a particle that has a different effective mass for inand out-side of the ellipsoid

$$
\begin{aligned}
& {\left[\frac{x^{2} J_{m}(x)}{x J_{m+1}(x)-m J_{m}(x)}\right]_{x=\sqrt{2 \lambda_{\text {in }}\left(\xi^{*}-1\right)}}} \\
& =\frac{2 \lambda_{\text {in }}}{\alpha R}\left[\frac{1}{y}+\frac{m_{\text {in }}}{m_{\text {out }}}\left(1-\frac{m+1}{y}+\chi_{\mu, m / 2}(y)\right)\right]_{y=\alpha R\left(\xi^{*}-1\right)}^{-1},
\end{aligned}
$$

where $\chi_{\mu, m / 2}(y)$ is defined by Eq. (29) and $\alpha, \lambda_{\text {in }}$ and $\mu$ depend on $m_{\text {out }}$. In the case when $m_{\text {in }}=m_{\text {out }}=m_{0}$ (34) becomes Eq. (28).

\section{Results of calculation and discussion}

In this section we present two sets of energy levels of a charged particle trapped in a potential well of strongly prolate ellipsoidal shape. The first set corresponds to an infinite potential well, while the second one is calculated for a finite potential well. The comparison of these sets of energy levels enables us to explore the influence of the depth of the potential 
well on the behaviour of the energy levels. Moreover, we present the energy levels as a function of the depth of the potential and reveal the region of validity of Eq. (31). To compare our results with numerical solutions for the infinite potential well [19] in calculations below we use the same set of parameters. As it is seen from Eq. (26) the

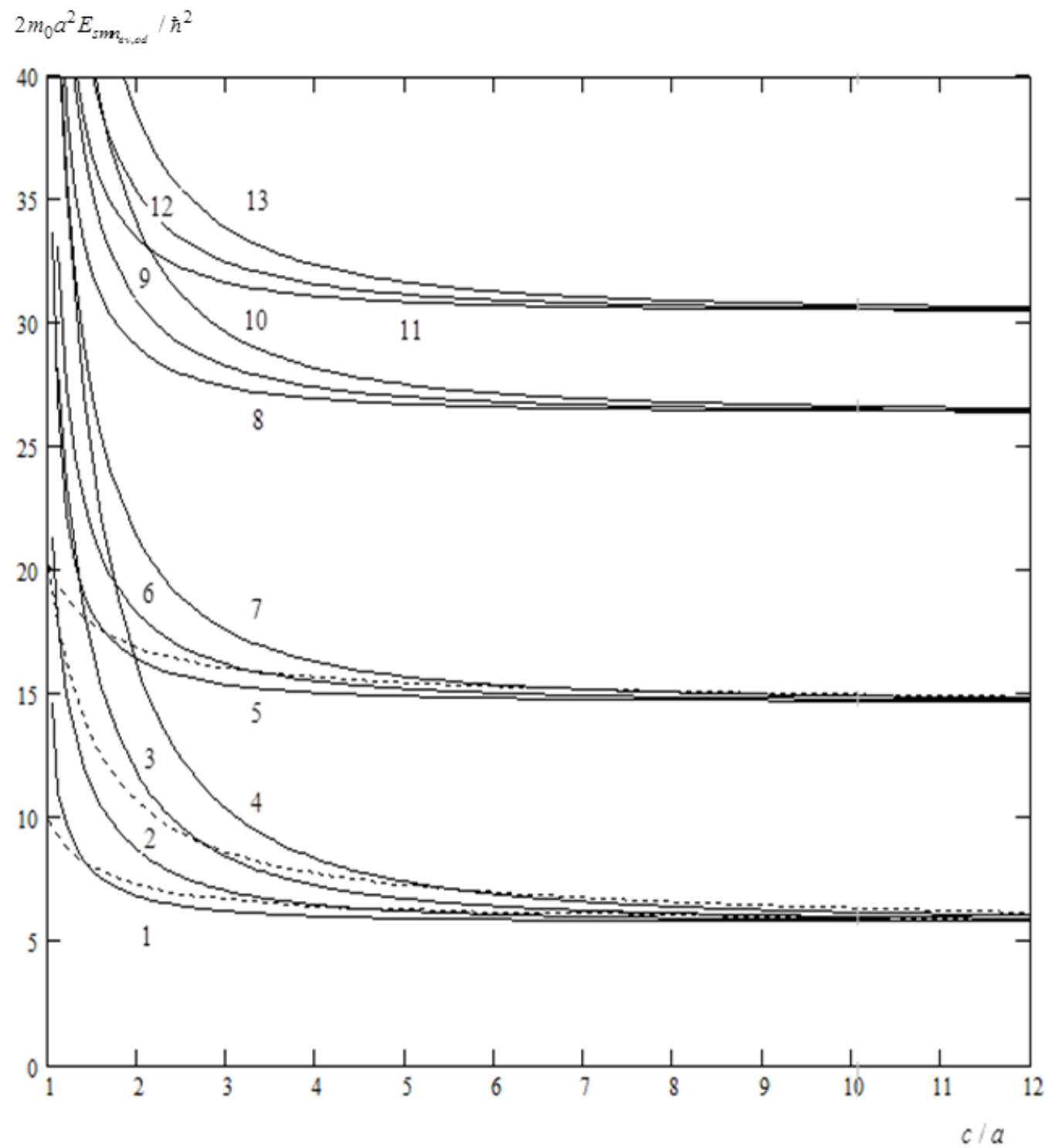

Fig. 2. Prolate ellipsoidal nanoparticle energy spectrum for the infinite potential well as a function of $c / a$. The solid curves - Eq. (26), the dotted curves - numerical solution of Eqs. (9a) and (9b) from Ref. [19]. The energies are shown in units $\hbar^{2} /\left(2 m_{0} a^{2}\right)$. Legend: curves $1-(101)$, curves $2-(102)$, curve 3 - (103), curve 4 - (104), curves 5 - (111), curve 6 - (112), curve 7 - (113), curve 8 - (121), curve 9 - (122), curve 10 - (123), curve 11 - (201), curve 12 - (202), curve 13 - (203) .

eigenenergy of a charged particle, in units of $\left(\hbar^{2} / 2 m_{0} a^{2}\right)$, depend only on the ratio $c / a$ but not on $a$ or $c$ separately. Moreover, the value $E_{n m n_{e v, o d}}^{0} /\left(\hbar^{2} / 2 m_{0} a^{2}\right)$ does not depend on $m_{0}$. Hence for a particle trapped in the infinite potential well, the behavior of energy levels given by (26) in units $\hbar^{2} /\left(2 m_{0} a^{2}\right)$ is universal and valid for nanoparticles made of arbitrary materials. When the potential well is finite and the particle's effective-mass is a different 
constant inside and outside of the ellipsoid, in (31) $E_{s m n_{e v, o d}}^{0}$ is given by Eq. (26), where $m_{0}=m_{\text {in }}$ and $\Omega_{\text {smn }}$ iv,od is defined by Eq. (30), where $m_{0}=m_{\text {out }}$.

Figure 2 shows the energy levels for the infinite ellipsoidal potential well calculated using Eq. (26). The states of a charged particle are specified with quantum numbers $s, m$ and $n_{e v, o d}$. As it is seen from Fig. 2 the states with the same $s$ and $m$ but different $n_{e v, o d}$ become almost degenerate at large values of $c / a$. An explanation follows: if at a fixed $a$ one increases $c$, each ellipsoidal confined state alters continuously in such a way that its limit is just a cylindrical quantum wire state having the same $m$ and the same number of nodes of the wave function along the "radial" coordinate [19,21,25]. Thus, all ellipsoidal quantum states with the same $s$ and $m$ but different $n_{e v, o d}$ create a miniband of the cylindrical quantum wire. Another peculiarity that follows from Fig. 2 is the appearance of levels crossings. The levels crossings occur because the energy levels with the same symmetry are differently ordered in the spherical quantum dot [9,21], and in the nanoparticle of strongly prolate ellipsoidal shape. For all values of $c / a$, the energy level corresponding to the even state is lying lower than the energy level corresponding to the odd state. For comparison, in Fig. 2 are shown the energy levels obtained in [19] by the numerical solution of Eqs. (9a) and (9b). The curves are plotted using the interpolation formulae for the energy levels $E_{101}, E_{102}$ and $E_{111}$ given in [19]. As it is seen from Fig. 2 for large $c / a$ the energy levels obtained with expression (26) are in good qualitative and quantitative agreement with the results obtained in [19].

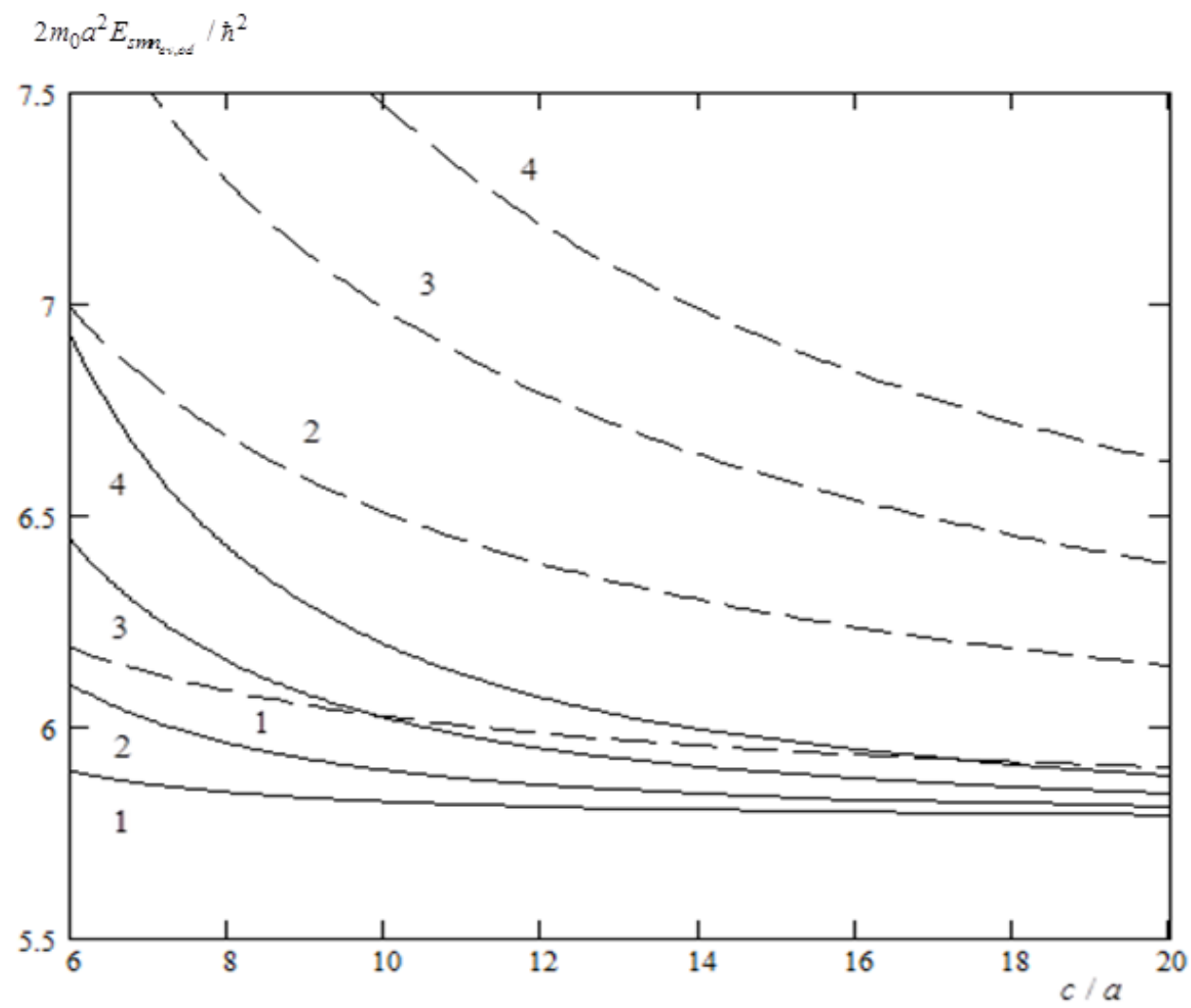

Fig. 3. The same as in Fig. 2 but for the energy levels with $s=1, m=0$ and $n_{e v}=1,3, n_{o d}=2,4$. The solid curves - Eq. (26), the dashed curves - Eq. (32). Legend: curves 1 - (101), curves 2 - (102), curves 3 - (103), curves $4-(104)$. 
In Fig. 3 are shown energy levels with $s=1, m=0$ and $n_{e v}=1,3, n_{o d}=2,4$ calculated using Eqs. (26) and (32) for large $c / a$. The comparison shows that the energy levels calculated with Eq. (32) significantly differ from the levels obtained in the present work and calculated in $[19,21]$. According to (26) a difference between the neighbour energy levels that create a miniband increases: $E_{s m 2}-E_{s m 1}=3 \Delta, E_{s m 3}-E_{s m 2}=5 \Delta, E_{s m 4}-E_{s m 3}=7 \Delta, \ldots$ where $\Delta=\pi^{2} \hbar^{2} /\left(8 m_{0} c^{2}\right)$, which is in a contradiction with (32).

When the potential well is finite the number of energy levels becomes finite. Thus, for $V_{0}<\infty$, the transcendental Eqs. (28) and (34) have a finite number of roots. In our calculations the employed values for $V_{0}$ and for the effective-mass are close to the characteristics of an electron in Gallium nitride GaN/AlN crystalline semiconductor nanoparticles [35,36]. The values of the effective masses $m_{i n}=0.18 m_{e}$ for $\mathrm{GaN}$ and $m_{\text {out }}=0.27 m_{e}$ for AIN ( $m_{e}$ is the mass of an electron) are obtained in Ref. [36] using the first-principles calculations of effective-mass parameters. In our calculations we use the around values for the effective masses: $m_{\text {in }}=0.2 m_{e}$ and $m_{\text {out }}=0.3 m_{e}$. At the first step, we perform calculations within the framework of the limited model under the assumption that the effective-mass of the particle is the same $m_{\text {in }}=m_{\text {out }}=0.2 m_{e}$ in- and out-side of the ellipsoid, which are presented in Figs. 4 and 5.

In Fig. 4, we present the four lowest energy levels of a charged particle confined in a finite potential well $V_{0}=1.5 \mathrm{eV}$ of the ellipsoidal shape when the small semi-axes of the ellipsoid is $a=1 \mathrm{~nm}$. The numerical calculation shows that Eq. (28) has root when $m=0$ and $s=1$ that create a miniband with different $n_{e v}$ and $n_{o d}$. So the motion perpendicular to the ellipsoidal axis leads to the existence of one bound level, while the number of levels related to the parallel motion is defined with the condition $E_{10 n_{e v, o d}}<V_{0}$. Figure 4 shows that for large $c / a$, the behavior of energy levels for the finite potential well is similar to the behavior of levels for the infinite potential well: the energy levels creating a miniband are simply shifted down. For our case the shift of energy levels is approximately 1.7 in units $\hbar^{2} / 2 m_{i n}^{*} a^{2}$.

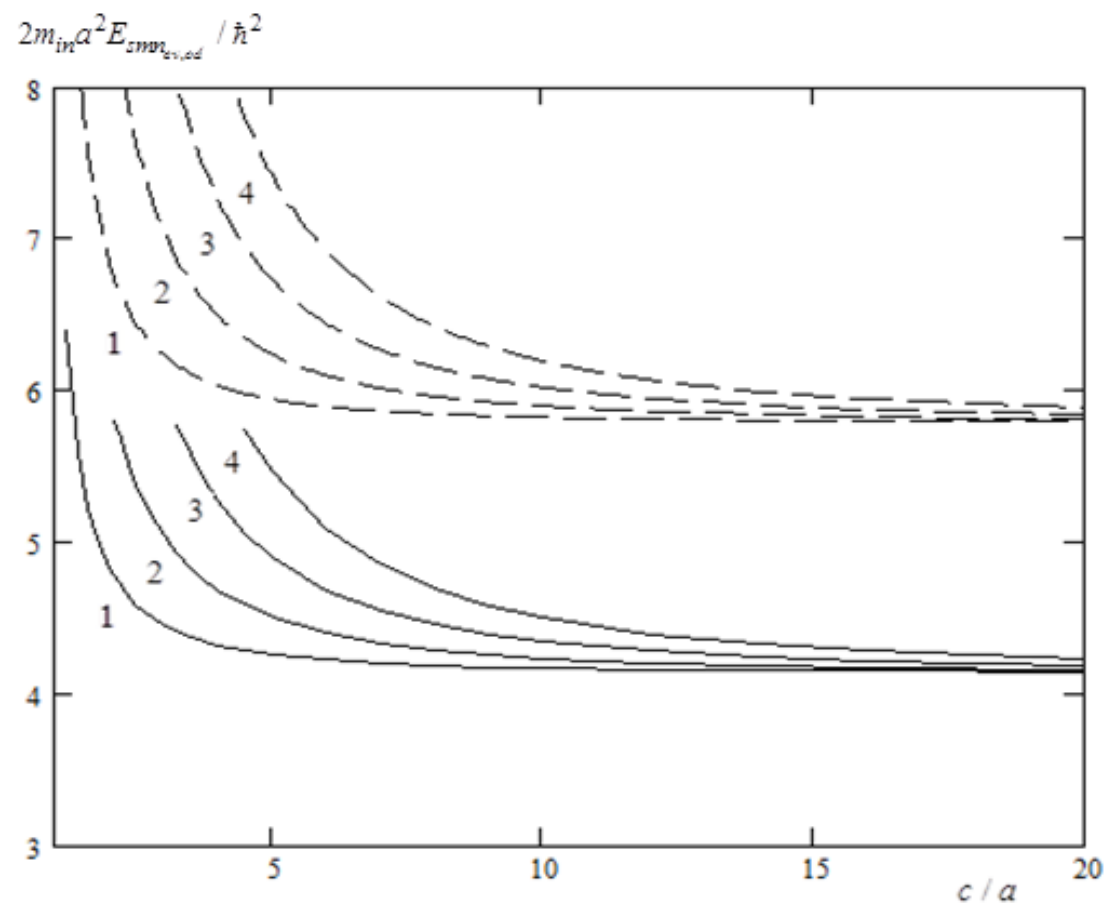


Fig. 4. Energy levels of prolate ellipsoidal nanoparticle as a function of $c / a$. The solid curves are results of calculations for the finite potential well $V_{0}=1.5 \mathrm{eV}$ using Eq. (28). The dashed curves are results of calculations for the infinite potential well using Eq. (26). The energies are shown in units $\hbar^{2} /\left(2 m_{i n} a^{2}\right)$. Small semi-axis $a=1 \mathrm{~nm}$. Legend: curves $1-(101)$, curves $2-(102)$, curves $3-(103)$, curves $4-(104)$.

In Fig. 5 are shown the ground and first excited state energy levels as a function of $V_{0}^{-1 / 2}$. The energy levels are obtained by means of a numerical solution of (28) and the approximate formula (31) for fixed values of the ellipsoidal semi-axes. The results of calculations clearly display that the energy levels calculated with (28) and (31) are close at

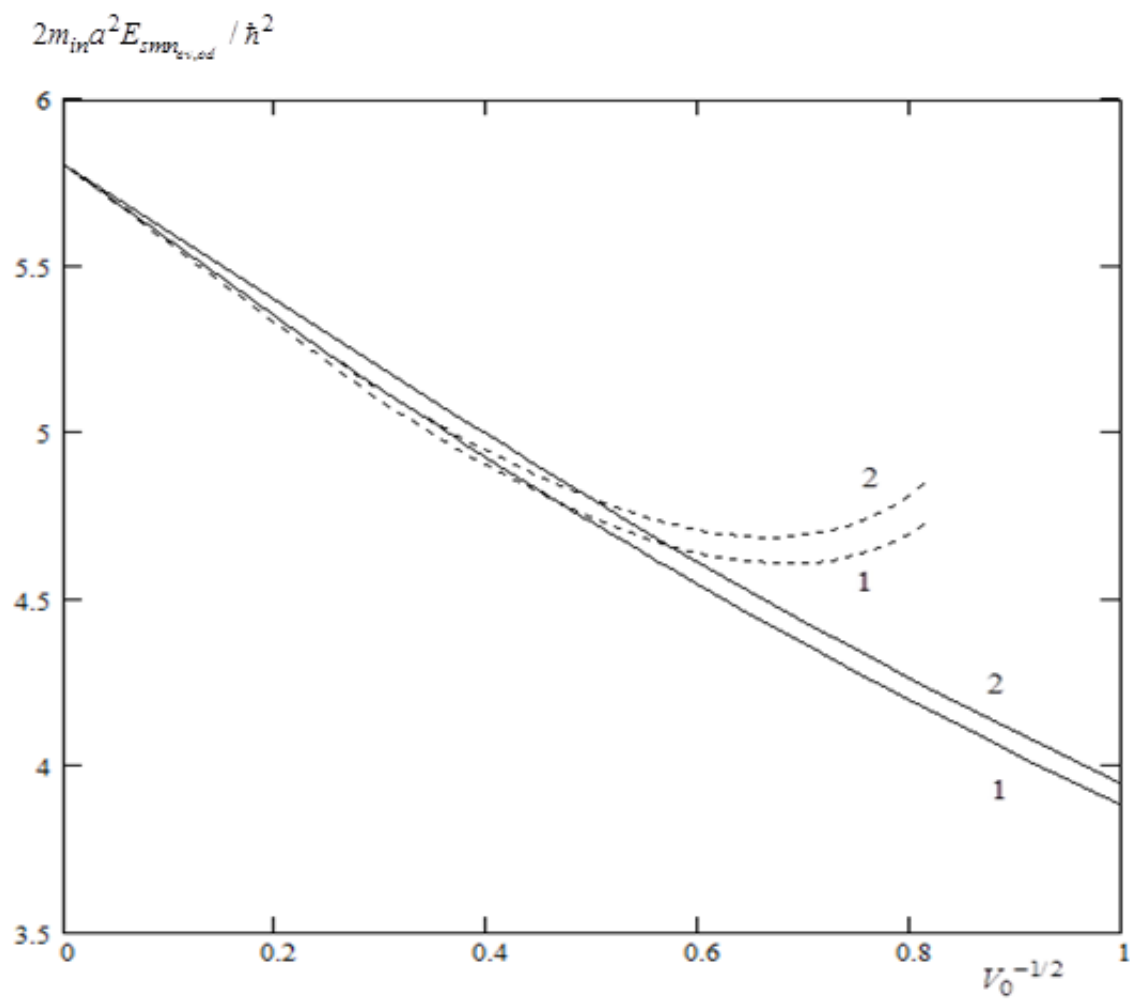

Fig. 5. The ground and first excited state energy levels as a function of $V_{0}^{-1 / 2}$ calculated using Eq. (28) (solid curves) and Eq. (31) (dotted curves) for $m_{\text {in }}=m_{\text {out }}=0.2 m_{e}$ and $a=1 \mathrm{~nm}, c=10 \mathrm{~nm}$. The energies are shown in units $\hbar^{2} /\left(2 m_{i n} a^{2}\right), V_{0}$ is given in $e V$. Legend: curves 1 - (101), curves $2-(102)$.

small $V_{0}^{-1 / 2}$ and linearly depend on $V_{0}^{-1 / 2}$. The comparison of the energy levels revealed that the region of validity of the approximate formula (31) is quite wide. For instance, in the calculated case of the ellipsoidal semi-axes, (31) correctly reproduces the behavior of ground state energy level for the potential well with $V_{0}>4 \mathrm{eV}$. 


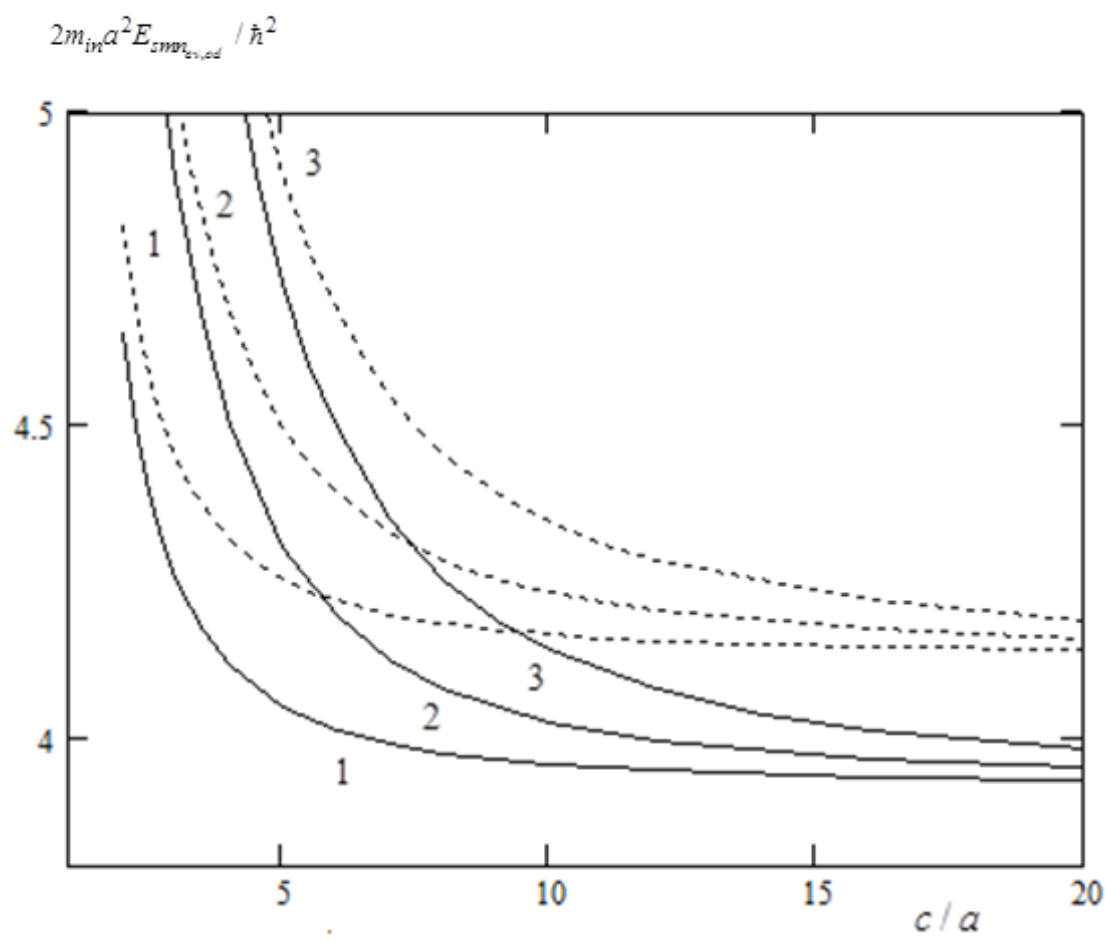

Fig. 6. The ground, first and second excited state energy levels as a function of $c / a$ calculated using Eq. (34) with $m_{i n}=0.2 m_{e}$ and $m_{\text {out }}=0.3 m_{e}$ (solid curves) and Eq. (28) with $m_{\text {in }}=m_{\text {out }}=0.2 m_{e}$ (dotted curves) for $a=1 \mathrm{~nm}$ and $V_{0}=1.5 \mathrm{eV}$. The energies are shown in units $\hbar^{2} /\left(2 m_{i n} a^{2}\right)$. Legend: curves $1-(101)$, curves $2-(102)$, curves 3 - (103) .

Now we consider the results obtained in the generalized model when the effective mass of a particle inside and outside of the ellipsoid is different. In Fig. 6, we present the energy levels calculated for an electron confined in the potential well of ellipsoidal shape using Eq. (34). The depth of the potential well is taken to be $V_{0}=1.5 \mathrm{eV}$, the effective mass of the electron is $m_{\text {in }}=0.2 m_{e}$ inside of the ellipsoid and $m_{\text {out }}=0.3 m_{e}$ outside of the ellipsoid. For the comparison we also present the results obtained under the assumption of equal masses: $m_{\text {in }}=m_{\text {out }}=0.2 m_{e}$. Figure 6 shows that when $m_{\text {in }} \neq m_{\text {out }}$ the energy levels are slightly shifted down. It is important to mention that our numerical calculations revealed the existence of the additional energy levels with $m=1, s=1$ and $n_{e v}=1,3, \ldots, n_{o d}=2,4, \ldots$ when $m_{\text {in }} \neq m_{\text {out }}$. This miniband of energy levels is located about six units above the energy levels with $m=0$ and $s=1$ and is not shown in Fig. 6. Thus unlike to the case $m_{\text {in }}=m_{\text {out }}$, the motion perpendicular to the ellipsoidal axis leads to the existence of two bound levels with $m=0,1$ and $s=1$.

\section{Conclusion}

The Schrödinger equation is solved for a strongly prolate ellipsoidal shaped potential well using the asymptotic approach, and the eigenfunctions and eigenvalues for a charged particle confined in this potential well are obtained. The eigenvalues are obtained for the infinite as well as finite potential wells. It is demonstrated that the traditional method of 
obtaining eigenvalues on matching the wave function and its derivative is valid when the potential well has a strongly prolate ellipsoidal shape and a finite depth. The formalism is generalized for the case when particle is described by the effective-mass Schrödinger equation when the particle's effective-mass is a different constant inside and outside of the ellipsoid. The calculated results are in good qualitative and quantitative agreement with the results obtained by previous authors $[19,21]$. The algebraic expressions have been derived for the energy spectrum of ellipsoidal nanoparticles for large $c / a$. The obtained expressions, together with the expression derived in our recent paper [21] for the potential well with a small deviation from the spherical symmetry, allow us to estimate the energy spectrum of ellipsoidal nanoparticles in a wide range of $c / a$.

\section{Acknowledgments}

This work was supported by Shota Rustavely Georgian National Science Foundation under the Grant DI/27/6-265/13.

\section{Appendix A}

Let us consider a charged particle trapped in a cylinder of length $L=2 c$ and radius $a$. The potential is zero inside the cylinder and is a constant $V=V_{0}>0$ on the surface of cylinder and outside (Fig. 1). Introducing cylindrical coordinates $\rho, z, \varphi$ and presenting the wave function of a charged particle as a product of three functions $\psi=R(\rho) \Phi(z) \exp ( \pm i m \varphi)$, one can obtain equations that describe motions along the cylinder axis $(-L / 2 \leq z \leq L / 2)$ and perpendicular to this axis $(0 \leq \rho \leq a)[25]$

$$
\begin{aligned}
& \frac{d^{2} \Phi_{i n s}}{d z^{2}}+k_{z}^{2} \Phi_{i n s}=0 . \\
& \frac{1}{\rho} \frac{d}{d \rho}\left(\rho \frac{d R_{i n s}}{d \rho}\right)+\left(k_{\rho}^{2}-\frac{m^{2}}{\rho^{2}}\right) R_{i n s}=0 .
\end{aligned}
$$

Here $k_{z}$ and $k_{\rho}$ are the separation parameters that are related by the condition $k_{z}^{2}+k_{\rho}^{2}=k^{2}$.

On the surface of cylinder and outside of it $\beta>0$, and in this domain the wave functions $\Phi_{\text {out }}(z)$ and $R_{\text {out }}(\rho)$ are solutions of equations

$$
\begin{aligned}
& \frac{d^{2} \Phi_{\text {out }}}{d z^{2}}-\left(\beta_{z}^{2}-k_{z}^{2}\right) \Phi_{\text {out }}=0, \\
& \frac{1}{\rho} \frac{d}{d \rho}\left(\rho \frac{d R_{\text {out }}}{d \rho}\right)-\left(\beta_{\rho}^{2}-k_{\rho}^{2}+\frac{m^{2}}{\rho^{2}}\right) R_{\text {out }}=0,
\end{aligned}
$$

where the separation parameters $\beta_{z}$ and $\beta_{\rho}$ are bounded with the condition $\beta_{z}^{2}+\beta_{\rho}^{2}=\beta^{2}$. We assume that a charged particle is strongly confined inside the cylinder, so the condition $\beta^{2}>k^{2}$ becomes fulfilled.

Eq. (A.1) has two solutions: the even one - $\Phi_{i n s}^{e v}(z)=\operatorname{cok}_{z} z$ and the odd one $\Phi_{\text {ins }}^{o d}(z)=\sin \left(k_{z} z\right)$ with respect to the replacement $z \rightarrow-z$. In the domains left of $-L / 2$ and right of $L / 2$, (A.3) has exponentially decreasing solutions: $\Phi_{\text {out }}=\exp \left( \pm \sqrt{\beta_{z}^{2}-k_{z}^{2}} z\right)$. On matching the inside and outside wave functions and their derivatives at the discontinuity of the potential, i.e. at $z= \pm L / 2$, we obtain the following equations: 


$$
\begin{gathered}
\operatorname{tg}\left(t_{e v}\right)=\sqrt{\left(\frac{L \beta_{z}}{2 t_{e v}}\right)^{2}-1,} \\
\operatorname{ctg}\left(t_{o d}\right)=-\sqrt{\left(\frac{L \beta_{z}}{2 t_{o d}}\right)^{2}-1},
\end{gathered}
$$

where $t_{e v, o d}=k_{z} L / 2$. Denoting the $n t h$ roots of transcendental Eqs. (A.5) and (A.6) as $t_{e v_{n}}$ and $t_{o d_{n}}$, respectively, for the separation parameter we write $k_{z_{e v}}=2 t_{e v_{n}} / L$ for the even and $k_{z_{o d}}=2 t_{o d_{n}} / L$ for the odd states. When the potential is high but finite, i.e. when $\beta_{z}^{2}=\beta_{\rho}^{2}=\beta^{2} / 2>>k^{2}$, for the approximate solutions of Eqs. (A.5) and (A.6) one can write

$$
t_{e v_{n}, o d_{n}}=\frac{\pi}{2} n_{e n, o d}\left(1-\frac{\hbar}{c \sqrt{m_{0} V_{0}}}\right),
$$

where $n_{e v}=1,3,5, \ldots$ and $n_{o d}=2,4,6, \ldots$.

The solution of Eq. (A.2), which is regular as $\rho \rightarrow 0$, is the Bessel function of the first kind $R_{i n s}=J_{m}\left(k_{\rho} \rho\right)$ [28], whereas the solution of Eq. (A.4), which is regular as $\rho \rightarrow \infty$, is the Macdonald function $R_{\text {out }}=K_{m}\left(\sqrt{\beta_{\rho}^{2}-k_{\rho}^{2}} \rho\right)$ [28]. By equating these wave functions and their derivatives on the surface of cylinder, i.e. at $\rho=a$, one gets [26]

$$
\left(\frac{1}{J_{m}\left(k_{\rho} \rho\right)} \frac{d J_{m}\left(k_{\rho} \rho\right)}{d \rho}\right)_{\rho=a}=\left(\frac{1}{K_{m}\left(\sqrt{\beta_{\rho}^{2}-k_{\rho}^{2}} \rho\right)} \frac{d K_{m}\left(\sqrt{\beta_{\rho}^{2}-k_{\rho}^{2}} \rho\right)}{d \rho}\right)_{\rho=a} .
$$

This transcendental equation determines a separation parameter $k_{\rho}$ that is related to the motion perpendicular to axis $z$; hence $k_{\rho}=\sigma_{s, m} / a$ with $\sigma_{s, m}$ the dual-index sth root of Eq. (A.8).

We obtain the energy spectrum of a charged particle confined in the cylinder through the equation $k_{\rho}^{2}+k_{z}^{2}=2 m_{0} E / \hbar^{2}$

$$
E_{s m n_{e v, o d}}=\frac{\hbar^{2}}{2 m_{0}}\left[\frac{\sigma_{s, m}^{2}}{a^{2}}+\left(\frac{2 t_{e v_{n}, o d_{n}}}{L}\right)^{2}\right],
$$

where $L=2 c$.

Thus, the energy of a particle confined to a cylinder is represented as a sum of two terms: the first term reflects the radial contribution to the total energy (the motion perpendicular to the cylinder axis), while the second term represents the small longitudinal energy contribution. The solution of Eq. (A.8) assumes an especially simple form when a depth of the potential well increases indefinitely. In that case for the energy spectrum, we have [25]

$$
E_{s m n_{e v, o d}}=\frac{\hbar^{2}}{2 m_{0}}\left[\frac{\tau_{s, m}^{2}}{a^{2}}+\left(\frac{\pi n_{e v, o d}}{2 c}\right)^{2}\right] .
$$

In (A.10) $\tau_{s, m}$ denotes the roots of Eq. (A.8) when $V_{0}=\infty$, i.e. $\tau_{s, m}$ are the roots of the Bessel function $J_{m}\left(\tau_{s, m}\right)=0$, and $t_{e v, o d}=\frac{\pi}{2} n_{e v, o d}$ are the roots of Eqs. (A.5) and (A.6) when $V_{0}=\infty$. In [25] the origin of the coordinate system is not located at the center of cylinder. Therefore, there are no the even and odd eigenstates (see Eq. (7. 27) in Ref. [25]). 


\section{Appendix B}

The following identical transformations are used throughout this paper [37]:

$$
\begin{aligned}
& \frac{1}{1-x / 2 q R}=1+\frac{x / 2 q R}{1-x / 2 q R}, \\
& \frac{1}{(1-x / 2 q R)^{2}}=1+\frac{x / q R-(x / 2 q R)^{2}}{(1-x / 2 q R)^{2}},
\end{aligned}
$$

where $q$ is a real or complex value.

\section{References}

1. P. Harrison, Quantum Well, Wires and Dots, Wiley, New York, 2005.

2. Al. L. Efros, A. L. Efros, Sov. Phys. Semicond. 16 (1982) 772.

3. X. G. Peng, L. Manna, W. Yang, J. Wickham, E. Scher, A. Kadavanich, and A. P. Alivisator, Nature 404 (2000) 59.

4. V. F. Puntes, K. M. Krishnan, and A. P. Alivisator, Science, 291 (2001) 2115.

5. L. S. Li, J. Hu, W. Yang, and A. P. Alivisator, Nano Lett. 1 (2001) 349.

6. J. Hu, L. S. Li, W. Yang, L. Manna, L.W. Wang, and A. P. Alivisator, Science, 292 (2001) 2060.

7. M. B. Mohamed, C. Burda and M. A. El-Sayed, Nano Lett. (2001) 589.

8. D. Tobia, E. De Biasi, M. Granada, H. E. Troiani, G. Zampieri, E. Winkler, and R. D. Zysler, J. Appl. Phys. 106 (2009) 103920.

9. A. B. Migdal 1959, in book of L. D. Landau, E. M. Lifshitz, Quantum Mechanics: NonRelativistic Theory, Elsevier, Singapore, 2007.

10. Al. L. Efros and A.V. Rodina, Phys. Rev. B 47 (1993) 10005.

11. K. Warda, Journal of Nanoscience and Nanotechnology 12 (2012) 284.

12. V. P. Dziuba, Yu. N. Kulchin, V. A. Milichko, Fiz. Tverd. Tela 56 (2014) 355.

13. K. G. Dvoyan, D. B. Hayapetyan, E. M. Kazaryan, A. A. Tshantshapanyan, Nanoscale Res. Lett. 2 (2007) 601.

14. K. G. Dvoyan, D. B. Hayrapetian, E. M. Kazaryan, Nanoscale Res. Lett. 4 (2009) 106.

15. A. A. Gusev, O. Chuluunbaatar, S. I. Vinitsky, K. G. Dvoyan, E. M. Kazaryan, H. A. Sarkisyan, V. L. Debrov, A. S. Klobotskaya, V. V. Serov, Yad. Fiz. 75 (2012) 1281.

16. T. Kereselidze, T. Tchelidze and T. Nadareishvili, http://arxiv.org/abs/1311.5977

17. G. Cantele, D. Ninno, G. Iadonisi, Nano Lett. 1 (2001) 121.

18. G. Cantele, D. Ninno, G. Iadonisi, Phys. Rev. B 64 (2001) 125325.

19. G. Cantele, G. Piacente, D. Ninno, G. Iadonisi, Phys. Rev. B 66 (2002) 113308.

20. H. Leon, J. L. Marin, R. Riera, Physica E 27 (2005) 385.

21. T. Kereselidze, T. Tchelidze, R. Ya. Kezerashvili, Physica E 68 (2015) 65.

22. M. van den Broek, F.M. Peeters, Physica E 11 (2001) 345.

23. L. C. Lew Yan Voon, C. Galeriu, M. Willatzen, Physica E 18 (2003) 547.

24. A. Bagga, S. Ghosh and P. K. Chattopadhyay, Nanotechnology, 16 (2005) 2726.

25. M. Kuno, Introductory Nanoscience, Garland Science, London, New York, 2012.

26. X. Leyronas, M. Combescot, Solid State Commun. 119 (2001) 631.

27. L. D. Landau, E. M. Lifshitz, Quantum Mechanics: Non-Relativistic Theory, Elsevier, Singapore, 2007.

28. E. Janke, F. Emde and F. Lösch, Tafeln Höherer Funktionen, B. G. Taubner Verlagsgesellschaft, Stuttgart (1960).

29. H. Bateman and A. Erdelyi, Higher Transcendental Functions, Mc Graw-Hill Book Company ING (1953).

30. D. L. Smith and C. Mailhiot, Rev. Mod. Phys. 62 (1990) 173.

31. A. T. Money, Brisire Gonul, E. P. O'Reilly, Phys. Rev. B 50 (1994) 10893.

32. E. A. Johnson, in Low-dimensional semiconductor structures. Fundamentals and device applications. Ed.: K. Barnham and D. Vvedensky. Cambridge University Press, 2001.

33. D. J. BanDaniel and C. B. Duke, Phys. Rev. 152 (1966) 683.

34. G. Bastard, Wave Mechanics Applied to Semiconductor Heterostructures. Halstead, 
New York, 1988.

35. A. N. Westmeyer, S. Mahajan. K. K. Bajaja, J. Y. Lin, H. X. Jiang, D. D. Koleske, and R.T. Senger, J. Appl. Phys. 99 (2006) 013705.

36. M. Suzuki, T. Uenoyama, A. Yanase, Phys. Rev. B 52 (1995) 8132.

37. T. M. Kereselidze, Z. S. Machavariani, and I. L. Noselidze, J. Phys. B: At. Mol. Opt. Phys. 31 (1998) 15. 